\title{
MATHEMATICAL
}

PROCEEDINGS

(formerly Proceedings)

of the

Cambridge Philosophical Society

VOLUME 118

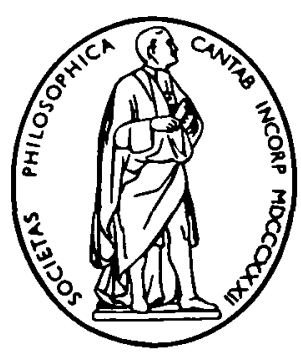


Published by the Press Syndicate of the University of Cambridge

The Pitt Building, Trumpington Street, Cambridge CB2 1RP 40 West 20th Street, New York, NY 10011-4211, USA

10 Stamford Road, Oakleigh, Melbourne 3166, Australia

(C) Cambridge Philosophical Society 1995

Printed in Great Britain by the University Press, Cambridge 


\section{INDEX FOR VOLUME 118}

Arason, J. \& Magnus, R. The universal multiplicity theory for analytic operator-valued functions.

Austin, D. M. \& Braam, P. J. Equivariant homology .

Bak, A. \& Vavilov, N. Normality for elementary subgroup functors .

Barrett, J. W. \& Westbury, B. W. The equality of 3-manifold invariants

Benson, D. J., Carlson, J. F. \& Rickard, J. Complexity and varieties for infinitely generated modules.

Blache, R. An example concerning Alexeev's boundedness results on log surfaces . . 65

Braam, P. J. \& Austin, D. M. Equivariant homology . . . . . . . . . . . 125

Bruce, J. W. Lines, circles, focal and symmetry sets . . . . . . . . . . . . . . 411

Bruns, W. \& Herzog, J. On multigraded resolutions . . . . . . . . . . . . 245

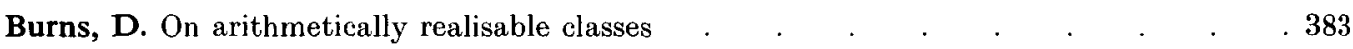

Carlson, J. F., Rickard, J. \& Benson, D. J. Complexity and varieties for infinitely generated modules

Casasayas, J., Llibre, J. \& Nunes, A. Periodic orbits of transversal maps . . . $\quad$. 161

Csörgö, S. \& Viharos, L. On the asymptotic normality of Hill's estimator . . . . 375

Cutler, C. D. Strong and weak duality principles for fractal dimension in Euclidean space 393

Dodds, P. G., Dodds, T. K., Dowling, P. N., Lennard, C. J. \& Sukochev, F. A. A uniform Kadec-Klee property for symmetric operator spaces . . . . . . . . . . 487

Dodds, T. K., Dowling, P. N., Lennard, C. J., Sukochev, F. A. \& Dodds, P. G. A uniform Kadec-Klee property for symmetric operator spaces

Dowling, P. N., Lennard, C. J., Sukochev, F. A., Dodds, P. G. \& Dodds, T. K. A uniform Kadec-Klee property for symmetric operator spaces

Duffield, N. G. \& O'Connell, N. Large deviations and overflow probabilities for the general single-server queue, with applications . . . . . . . . . . . . . . . 363

Elvira-Donazar, C. \& Hernandez-Paricio, L.-J. Closed model categories for the $n$-type of spaces and simplicial sets .

Grandis, M. Homotopical algebra and triangulated categories _ _ . . . . . . $\quad$. 259

Harman, G. Numbers badly approximable by fractions with prime denominator . . 1

Hernandez-Paricio, L.-J. \& Elvira-Donazar, C. Closed model categories for the $n$-type of

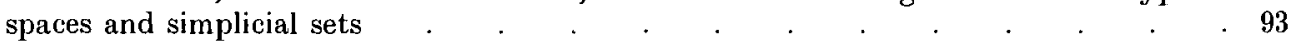

Herzog, J. \& Bruns, W. On multigraded resolutions . _ . . . . . . . . . . . . 245

Hofmann, K. H., Morris, S. A., Oates-Williams, S. \& Obraztsov, V. N. Locally compact groups with closed subgroups open and $p$-adic . . . . . . . . . . $\quad . \quad 303$

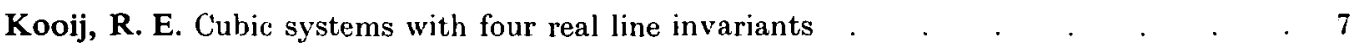

Kratz, W. An oscillation theorem for self-adjoint differential systems and an index result for corresponding Riccati matrix differential equations. . . . . . . . . . 351

Kuijlaars, A. B. J. \& Saff, E. B. Asymptotic distribution of the zeros of Faber polynomials 437

Lehrer, G. I. A toral configuration space and regular semisimple conjugacy classes . $\quad 105$

Lennard, C. J., Sukochev, F. A., Dodds, P. G., Dodds, T. K. \& Dowling, P. N. A uniform Kadec-Klee property for symmetric operator spaces . . . . . . . . . . 487

Letherman, S. D. \& Wood, R. M. W. A note on the Julia set of a rational function. . 477

Leung, D. H. Some stability properties of $\mathrm{C}_{\mathrm{o}}$-saturated spaces . . . . . . . . 287

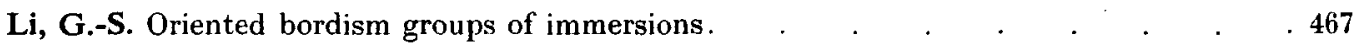


Lichtman, A. I. Algebraic elements in matrix ring over division algebras . . . . . 215

Llibre, J., Nunes, A. \& Casasayas, J. Periodic orbits of transversal maps . . . 161

Lyzzaik, A. Covering properties of open continuous mappings having two valences between Riemann surfaces . . . . . . . . . . . . . . . . 321

Magnus, R. \& Arason, J. The universal multiplicity theory for analytic operator-valued functions.

Menth, M. A family of Fitting classes of supersoluble groups . . . . . . . . . . . 49

Merkulov, S. A. Moduli spaces of compact complex submanifolds of complex fibered manifolds

Merriman, J. R. \& Smart, N. P. Curves of genus 2 with good reduction away from 2 with. a rational Weierstrass point (Corrigenda)

Montgomery, H. L., Vaughan, R. C. \& Wooley, T. D. Some remarks on Gauss sums associated with kth powers

Morris, S. A., Oates-Williams, S., Obraztsov, V. N. \& Hofmann, K. H. Locally compact groups with closed subgroups open and $p$-adic

Müller, E. F. Differential fields and differentiable functions of algebraic numbers $\quad . \quad 341$

Nunes, A., Casasayas, J. \& Llibre, J. Periodic orbits of transversal maps . . . 161

O'Connell, N. \& Duffield, N. G. Large deviations and overflow probabilities for the general single-server queue, with applications

Oates-Williams, S., Obraztsov, V. N., Hofmann, K. H. \& Morris, S. A. Locally compact groups with closed subgroups open and $p$-adic

Obraztsov, V. N., Hofmann, K. H., Morris, S. A. \& Oates-Williams, S. Locally compact groups with closed subgroups open and $p$-adic

Offord, A. C. The distribution of the values of an entire function whose coefficients are independent random variables (II) .

Popov, I. Yu. The operator extension theory, semitransparent surface and short range potential.

Rendall, A. D. Global properties of locally spatially homogeneous cosmological models with matter

Rickard, J., Benson, D. J. \& Carlson, J. F. Complexity and varieties for infinitely generated modules

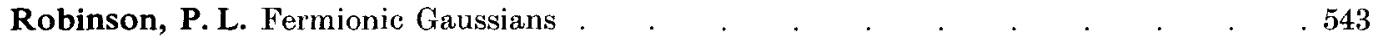

Saff, E. B. \& Kuijlaars, A. B. J. Asymptotic distribution of the zeros of Faber polynomials 437

Seeger, A., Wainger, S. \& Wright, J. Pointwise convergence of spherical means . $\quad .115$

Shirvani, M. The finite inner automorphism groups of division rings . . . . . . . . 207

Smart, N. P. \& Merriman, J. R. Curves of genus 2 with good reduction away from 2 with a rational Weierstrass point (Corrigenda)

Soma, T. A rigidity theorem for Haken manifolds . . . . . . . . . . . . . 141

Stöhr, R. Symmetric powers, metabelian Lie powers and torsion in groups. . . . . . 449

Sukochev, F. A., Dodds, P. G., Dodds, T. K., Dowling, P. N. \& Lennard, C. J. A uniform Kadec-Klee property for symmetric operator spaces . . . . . . . . . . $\quad .487$

Trofimov, V. I. \& Weiss, R. M. Graphs with a locally linear group of automorphisms $\quad 191$

Vaughan, R. C., Wooley, T.D. \& Montgomery, H. L. Some remarks on Gauss sums associated with kth powers

Vavilov, N. \& Bak, A. Normality for elementary subgroup functors . . . . . . . . 35

Viharos, L. \& Csörgö, S. On the asymptotic normality of Hill's estimator _ . . . . 375

Wainger, S., Wright, J. \& Seeger, A. Pointwise convergence of spherical means _ . 115

Weiss, R. M. \& Trofimov, V. I. Graphs with a locally linear group of automorphisms . 191

Westbury, B. W. \& Barrett, J. W. The equality of 3-manifold invariants . . . . . 503

Wood, R. M. W. \& Letherman, S. D. A note on the Julia set of a rational function. $\quad .477$ 
Wooley, T. D., Montgomery, H. L. \& Vaughan, R. C. Some remarks on Gauss sums associated with kth powers .

Wright, J., Seeger, A. \& Wainger, S. Pointwise convergence of spherical means $\quad$. $\quad 115$

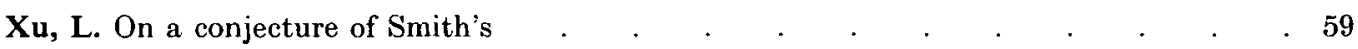

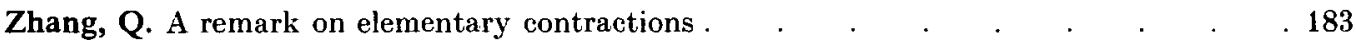




\section{THE PREPARATION OF MANUSCRIPTS}

\section{The attention of authors is particularly directed to the following requests.}

1. Papers should be typed, double-spaced, on one side of white paper (of which $\mathrm{A} 4,210$ by $297 \mathrm{~mm}$, is a suitable size). The pages must be numbered. Margins of $30 \mathrm{~mm}$ should be left at the side, top and bottom of each page. Two clear copies should be sent.

A cover page should give the title, the author's name and institution, with the address to which mail should be sent.

The title, while brief, must be informative (e.g. A new proof of the prime-number theorem, whereas Some applications of a theorem of G. H. Hardy would be useless).

The first paragraph or two should form a summary of the main theme of the paper, providing an abstract intelligible to mathematicians.

For a typescript to be accepted for publication, it must accord with the standard requirements of publishers, and be presented in a form in which the author's intentions regarding symbols etc, are clear to a printer (who is not a mathematician).

The following notes are intended to help the author in preparing the typeseript. New authors may well enlist the help of senior colleagues, both as to the substance of their work and the details of setting it out correctly and attractively.

\section{Notation}

Notation should be chosen carefully so that mathematical operations are expressed with all possible neatness, to lighten the task of the compositor and to reduce the chance of error.

For instance $n_{2}(n$ sub $k)$ is common usage, but avoid if possible using $c$ sub $n$ sub $k$. Fractions are generally best expressed by a solidus. Complicated exponentials like

should be shown in this and no other way.

$$
\exp \left\{z^{2} \sin \theta /\left(1+y^{2}\right)\right\}
$$

In the manuscript, italics, small eapitals and capitals are specified by single, double and triple underlinings. Bold faced type is shown by wavy underlining; wavy will be printed wavy.

It helps if displayed equations or statements which will be quoted later are numbered in order on the right of their line. They can then be referred to by, for example, 'from ( 7$)^{\text {'. }}$

The authòr must enable the printer (if necessary by pencilled notes in the margin) to distinguish between similar symbols such as $0,0,0,0,0 ; x, \mathrm{X}, \mathrm{X}: \phi, \Phi, \varnothing ; 1,1 ; \varepsilon, \in ; \kappa, k$.

Greek letters can be denoted by Gk in the margin.

If an author wishes to mark the end of the proof of a theorem, the sign I may be used.

Footnotes should be avoided.

\section{Diagrams}

It is extremely helpful if diagrams are drawn in Indian ink on white eard. faintly blue or green-lined graph paper, or tracing cloth or paper. Symbols, legends and captions should be given on a transparent overlay. Each text figure must be numbered as Figure 1, Figure 2, ... and its intended position elearly indicated in the manuscript:

\section{Figure 1 here}

The author's name in pencil must be on all separate sheets of diagrams.

A figure is expensive to reproduce and should be included only when the subject matter demands it, or when it greatly clarifies the exposition.

The Society recognizes that some authors do not have the facilities for producing drawings of a sufficiently high standard to be reproduced directly and it is therefore willing to have such diagrams redrawn, provided that they are clear.

\section{Tables}

Tables should be numbered (above the table) and set out on separate sheets. Indicate the position of each in the text as for figures:

Table 3 here

\section{References}

References should be collected at the end of the paper numbered in alphabetical order of the authors' names. Titles of journals should be abbreviated as in Mathematical Reviews. The following examples show the preferred style for references to a paper in a journal, a paper in a proceedings volume, a book and an unpublished dissertation:

[1] J. F. ADAMs. On the non-existence of elements of Hopf invariant one. Ann. of Math. (2) 72 (1960), 20-104.

[2] M. P. Fourman and D. S. Scotr. Sheaves and logic. In Applications of Sheaves, Lecture Notes in Math. vol. 753 (Springer-Verlag, 1979), pp. 302-401.

[3] P. T. Johnstone. Stone Spaces. Cambridge Studies in Advanced Math. no. 3 (Cambridge University Press, 1982).

[4] F. W. Lawvere. Functorial semantics of algebraic theories. Ph.D. thesis. Columbia University (1963). 


\section{Mathematical Proceedings of the Cambridge Philosophical Society}

MPCPCO 118 (Pt 3) 383-57 I (1995) 0305-0041 November 1995

\section{CONTENTS}

Burss, D. On arithmetically realizable classest

CuTr FR, C. D. Strong and weak duality principles for fractal dimension in Euclidean space.

BRUCE, J. W. Lines, cireles, focal and symmetry sets

Kuidlanis, A. B. J. \& SAFF, E. B. Asymptotic distribution of the zeros of Faber polynomials . 437

SröнR, R. Symmetric powers, metabelian Lie powers and torsion in groups . . . . . 449

LI, G,-S. Oriented bordism groups of immersions . . . . . . . . . . . 467

LETHERman, S. D. \& Wood, R. M. W. A note on the Julia set of a rational function . . . 477

Dopns, P. G., Dodns, T. K., Downeng, P. N., Lennard, C. J. \& Sukochev, F. A. A uniform Kadee-Klee property for symmetric operator spaces _ . . . . . . . . . . . 487

Barrett, J. W. \& Westbury, B. W. The equality of 3-manifold invariants. . . . . 503

RENDALL, A. D. Global properties of locally spatially homogeneous cosmological models with matter

OFFORD, A. C. The distribution of the values of an entire function whose coefficients are independent random variables (II)

Robrnson, P. L. Fermionic Gaussians

Popov, I. Yv. The operator extension theory, semitransparent surface and short range potential

Proceedings of Meetings .

(C) The Cambridge Philosophical Society 1995

Printed in Great Britain by the University Press, Cambridge

\section{CAMBRIDGE UNIVERSITY PRESS}

